\title{
WHAT SHALL WE TEACH CONCERNING THE PHYSIOLOG- ICAL EFFECTS OF ALCOHOL?
}

\author{
By $\cdot$ Harry Clifford Doane.
}

Editorial Note: This article will serve to remind us that a few years ago there was an abundance of evidence relative to the physiological effects of alcohol. But few people cared to consider it dispassionately then, owing to the obviously untrustworthy claims made by the chief exploiters of the evidence. Now, however, we may return with equanimity to the consideration of experimental data from all sources and ultimately hope to come at the whole truth.

The teaching of physiology and hygiene is far from being in a satisfactory condition in our schools. The agitation so vigcrously prosecuted" by some against the various state laws requiring the teaching of the effects of alcohol and narcotics and the criticisms of the accuracy of the statements on this subject found in our school physiologies seem to be in a measure responsible for a serious neglect of physiology teaching in many schools. There appears to have grown up a widespread feeling that physiology is an unfit subject for grade schools, and even for high schools unless it can be pursued in a thoroughly scientific manner by laboratory methods.

I do not wish to be understood as minimizing in any degree the value or need of making scientific subjects the means of teaching scientific methods of thought, nor would I decry the place of the laboratory in such instruction; but physiology occupies a peculiar position among the sciences as a subject of school study. It is essential to the physical welfare of the individual and of the community that the pupils of our schools acquire a knowledge of the more important principtes of hygiene. These principles of hygiene are dependent for their truth on the facts of physiology, and cannot be successfully taught without some reference to physiology as a basis. The value of a knowledge of physiology and hygiene for their own sake makes it important that they be tatight definitely and systematically in all schools, even though laboratory methods cannot be used, beginning as low in the grades as possible. Many facts of physiology and hygiene can be successfully taught in the first and second grades. 
The claim that place should be given in our teaching of physiology and hygiene to the effects of alcohol and narcotics needs no defense. In the year ending June 30,1907 , the people of the United States consumed 2,019,690,9 I I gallons of distilled spirits, wines and malt liquors, according to the "Statistical Abstract of the United States," published by the government. A careful estimate gives the retail cost of this liquor as $\$ 2,275$,070,857 . The per capita consumption was as follows:

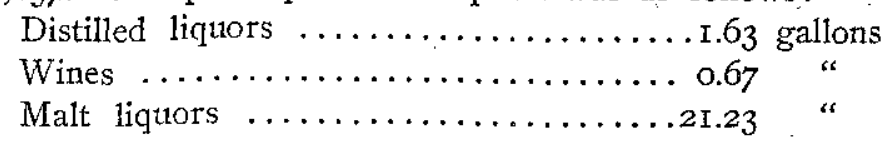

Total per capita ..............23.53

'This gives a per capita cost of $\$ 26.50$.

The people can be brought to get rid of this enormous, worse than useless, expense only by educating them as to the true facts in the case.

We who are not specialists in this subject must accept and teach the conclusions of those who have scientifically and experimentally investigated the effects of alcohol upon the human body and its processes. In the following paragraphs I have collected some of the testimony of such investigation.

Effect of Alcohol on Muscular Effort.--Professor Destree of Belgium measured the weight he could lift by flexing the middle finger. In six tests he could lift nearly seventeen pounds. After fatigue came on he could lift only nine pounds in the same number of tests. When he had taken some wine and rested thirty minutes, he could raise but three and one half pounds. During a period of ten years Professor Kraepelin performed some 3,000 experiments on the effects of alcohol. He found by means of a dynamometer that the pull that could be given with the hand was weakened by taking small quantities of alcohol.

Mountain climbers usually recommend that no alcoholic drinks be used just before or during the climb. Professor A. Durig reports the results of experiments performed in 1906 to determine the effect of alcohol on mountain climbing. Careful observation of the strbject of the experiment" in climbs when "2 to 2.5 fluid ounces of alcohol, equivalent to about a pint of wine, were taken before the ascent" and also when no alcohol was taken, showed that "alcohol diminished the amount of work performed per minute by about one-sixth." 
In "Physiological Aspects of the Liquor Problem," Professor Hodge of Clark University describes many of his own experiments showing the effect of alcohol on animals. He trained four selected puppies to recover a ball thrown across a gymnasitun. To two of the dogs he gave food mixed with dietetic doses of alcohol while the others were fed normally. The ball was thrown too feet as rapidly as recovered. This was repeated roo times each day for fourteen successive days. Out of the 1,400 times the dogs to which alcohol had been given brought back the ball only $47^{8}$ times, while the others secured it 922 times.

Dr. Parkes experimented with two gangs of men, selected to be as nearly similar as possible, in mowing. He found that with one gang abstaining from alcoholic drinks and the other not, the abstaining gang could accomplish more. On transpos. ing the gangs the same results were repeatedly obtained. Similar results were obtained by Professor Aschaffenburg of Heidelburg University, who found experimentally that men "were able to do 15 per cent less work after taking alcohol." Professor Abel of Johns Hopkins University says: "Both science and the experience of life have exploded the pernicious theory that alcohol gives any persistent increase of muscular power."

Alcohol and the Circulation.-As a tesult of experiments performed in 1869, Zimmerberg declares: "In the light of these experiments one is not only justified in denying to alcohol any stimulating power whatever for the heart, but, on the contrary, in cleclaring that it lowers the working capacity of that organ."

Dr. J. H. Kellogg, head of the Battle Creek Sanitaritm, says: "The full bounding pulse ustally produced by the administration of an ounce or two of brandy properly diluted; gives the impression of an increased vigor of heart action; but it is only necessary to determine the blood pressure by means of a RivaRocci instrument, or Gaertner's tonometer, to discover that the blood pressure is lowered instead of raised. This lowering may amount to twenty or thirty millimeters, or even more. *** $*$ It can readily be seen, then, that the bounding pulse is not the result of increased heart vigor, but indicates rather a weakened state of the heart, combined with a dilated condition of the small vessels."

In an address before the Liverpool Medical Association, Dr. James Barr, president of the association, discussing the effects 
of medicinal doses of alcohol upon the circulation remarked: "It causes dilatation of the arterioles and of all arteries well suppied with muscular fibers, owing to its paretic effect upon the vasomotor nervous system, and its direct action as a protoplasmic poison on the muscular fiber. It has a similar, though less marked, action on the cardiac muscle. From these causes the systolic blood pressure is lowered, the systolic output from the heart is diminished, and the cardiac energy is wasted in pumping blood into relaxed vessels; the large bounding pulse with comparatively short systolic period, which givés a deceptive appearance of vigor and force in the circulation, is due to the large wave in the dilated vessels."

Effects of Alcohol on the Nervous System.-In an article in the Journal of Inebriety, Dr. J. W. Grosvener of Buffalo says: "Alcohol is a paralyzer. The truth of this proposition has been demonstrated experimentally scores of times by world-famed physiologists. Says Forel: 'Through all parts of nervous aclivity from the innervation of the muscles and the simplest sensation of the highest activity of the soul the paralyzing effect of alcohol cain be demonstrated.' Several experimenters of undoubted ability have noted the paralyzing effect of alcohol even in small doses. By the use of delicate instruments of precision, Ridge tested the effect of alcohol on the senses of smell, vision, and muscular sense of weight. He found that two drams of absolute alcohol produced a positive decrease in the sensitiveness of the nerves of feeling, that so small a quantity as one half dram of absolute alcohol diminished the power of vision and the muscular sense of weight. Kraepelin and Kurz by experiment determined that the acuteness of the special senses of sight, hearing, touch, taste, and smell was diminished by an eunce of alcohol, the power of vision being lost to one third of its extent and a similar effect being produced on the other special senses. Other investigators, as Crothers, Madden, Kellogg, Frey, von Bunge have reached like conclusions."

Dr. Kellogg, previously quoted, in an article giving conclusions from experiments by himself and others says: "Kleefeld has shown that alcohol, when taken into the blood, produces almost immediately a marked change in the minute structures of the brain. The dendrons and contact globules are shriveled, thus breaking contact and thus interrupting the normal nerve circuits. This fact explains, to a large degree, the mental and 
moral effects of alcohol, especially its effect in destroying inhibition. When often repeated, this toxic effect gives rise to degenerative changes which are seen in their full development in general paresis. The flushing of the face seen after the administration of alcohol, and the exhilaration felt by a person in ordinary health, are due, not to a stimulating effect, but rather to a paralyzing or sedative effect. $* * *$ A person who is fatigued, after taking alcohol feels relieved, not because he is rested or because his muscles have been reinforced, but because the nerves of fatigue are paralyzed so that he no longer appreciates the fact that he is fatigued. A person who is cold, after taking a dram, no longer suffers from cold or chilliness, and imagines that the alcohol has warmed him. This is not the case, however. The alcohol has only lessened the sensibility of his thermic nerves, so that he is less sensitive to cold, while at the same time increasing the flow of blood to the skin by paralyzing the vasomotor centers."

With regard to the supposed quickening of the mental processes Horsley and Sturge, in their recent book, "Alcohol and the Human Body," say: "Kraepelin found that the simple reaction period, by which is meant the time occupied in making a mere response to a signal, as, for instance, to the sudden appearance of a flag, was, after the ingestion of a small quantity of alcohol ( $I / 4$ to $I / 2$ ounce), slightly accelerated; that there was, in fact, a slight shortening of the time, as though the brain were enabled to operate more quickly than before. But he found that after a few minutes, in most cases, a slowing of mental action began, becoming more and more marked, and enduring as long as the alcohol was in active operation in the body, i. e., four to five hours. *** Kraepelin found that it was only more or less automatic work, such as reading aloud, which was quickened by alcohol, though even this was rendered less trustworthy and accurate." Again: "Kraepelin had always shared the popular belief that a small quantity of alcohol (one to two teaspoonfuls) had an accelerating effect on the activity of his mind, enabling him to perform test operations, as the adding and subtracting and learning of figures more quickly. But when he came to measure with his instruments the exact period and time occupied, he found, to his astonishment, that he had accomplished these mental operations not more, but less, quickly than before. *** Numerous further experiments were 
carried out in order to test this matter, and these proved that alcohol lengthens the time taken to perform complex mental processes, while by a singular illusion the person experimented upon imagines that his psychical actions are rendered more rapid."

Relation of Digestion and Nutrition to Alcohol.-As a result of his own experiments Prof. Chittenden of Yale University remarks: "We believe that the results obtained justify the conclusion that gastric digestion as a whole is not materially modified by the introduction of alcoholic fluids with the food. In other words, the tnquestionable acceleration of gastric sectetion which follows the ingestion of alcoholic beverages is, as a rule, counterbalanced by the inhibitory effect of the alcoholic fluids upon the chemical process of gastric digestion, with perhaps at times a tendency towards preponderance of inhibitory action." Kellogg, Sir William Roberts and others have come to the same or stronger conclusions as to the undesirable action of alcohol on digestion, as a result of their own experiments.

Horsley and Sturge, in their book previously mentioned, say: "Hundreds of men and women who haunt the out-patient departments of hospitals suffer from chronic atony and slight dilatation of the stomach, which arise in part from the badly cooked food they eat, but chiefly owe their origin to the debilitating effect of alcohol upon the muscular walls of this organ and the fermentation of its retained contents."

The experiments of Professor Atwater demonstrated that a certain amount of alcohol is oxidized in the body. As the oxidation produces energy he held that alcohol was a food. To call alcohol a food is to distort the meaning of food as ordinarily used and few of the physiologists who can properly be considered authorities agree with Atwater in calling alcohol a food instead of a poison.

Kellogg points out that strychnine, quinine and many other drugs are oxidized in the body, but strely cannot be called foods. The following reasons for not considering alcohol a food are taken from Kellogg:

"I. A habitual user of alcohol has an intense craving for his accustomed dram. Without it he is entirely unfitted for business. One never experiences such an insane craving for bread, potatoes, or any other particular article of food. ***

"2. By continuous use the body acquires a tolerance for al- 
cohol. That is, the amount which may be imbibed and the amount reqtired to produce the characteristic effects first experienced gradually increase until very great quantities are sometimes required to satisfy the craving which its habitual use often produces: This is never the case with true foods. $* * *$ Alcohol behaves in this regard just as does opitum or any other drug. It has no resemblance to a food.

"3. When alcohol is withdrawn from a person who has been accustomed to its daily use, most distressing effects are experienced. *** Who ever saw a man's hand trembling or his nervous system unstrung because he could not get a potato or a piece of cornbread for breakfast? In this respect, also, alcohol behaves like opium, cocaine, or any other enslaving drug.

"4. Alcohol lessens the appreciation and the value of brain and nerve activity, while food reinforces nervous and mental energy.

"5. Alcohol as a protoplasmic poison lessens muscular power. wherẹas food increases energy and endurance.

" 6 . Alcohol lessens the power to endure cold. This is true to such a marked degree that its use by persons accompanying Arctic expeditions is absolutely prohibited. Food, on the other hand, increases ability to endure cold. The temperature after taking food is raised. After taking alcohol the temperature, as shown by the thermometer, is lowered.

"7. Alcohol cannot be stored in the body for future use, whereas all food substances can be so stored.

"8. Food burns slowly in the body, as it is required to satisfy the body's needs. Alcohol is readily oxidized and eliminated, the same as any other oxidizable drug."

The superintendent of the Northern Illinois Hospital for the Insane, Dr. Vaclav H. Podstata, says: "Alcohol is unquestionably a direct and powerful poison to the nervous structure. It not only temporarily impairs and prevents the activity of nervous tissue, but in a measure, though at times very minute and almost imperceptible, every dose of it permanently disables the functional activity of those organs."

(To be continued.) 